\title{
Long-term complications of modified Soave radical correction in the treatment of Hirschsprung's disease and its influences on life quality.
}

\author{
Zan Xi ${ }^{1,2}$, Lingyu Kong ${ }^{2}$, Yajun Chen ${ }^{1 *}$ \\ ${ }^{1}$ Department of Surgery, Beijing Children's Hospital Affiliated to Capital Medical University, Beijing, PR China \\ ${ }^{2}$ Emergency Department, First Affiliated Hospital of Xinxiang Medical University, PR China
}

\begin{abstract}
Objective: To investigate the long-term complications of modified Soave radical correction in the treatment of Hirschsprung's disease and its influences on life quality.

Method: 50 child patients with Hirschsprung's disease treated in our hospital from January 2014 to September 2017 were selected as objects of study and received modified Soave radical correction. The long-term complications, anus function and life quality of all patients were observed. TACQOL scale was used for life quality.

Results: There were 8 cases with long-term complications after operation, and the incidence was $16 \%$. The life quality scores of child patients were still significantly different compared with those of normal children $(P<\mathbf{0 . 0 5})$. Moreover, the excellent-good rate of anus function was up to $86 \%$.

Conclusion: In the treatment of Hirschsprung's disease, modified Soave radical correction can reduce the incidence rate of postoperative long-term complications, and help shorten the recovery time of defecation function and anus function after operation, thus gradually improving children's life quality.
\end{abstract}

Keywords: Modified soave radical correction, Hirschsprung's disease, Long-term complications, Life quality. Accepted on January 15, 2018

\section{Introduction}

Hirschsprung's Disease (HD) is a kind of aganglionosis, as well as a common anomaly of digestive tract in pediatric surgery, which is clinically characterized by the absence of ganglion cells in intestinal canal. Modified Duhamel radical correction is a common treatment means for HD [1].

In 1995, HD was successfully treated with laparoscopic Soave radical correction, and the success was obtained in the newborn [2].

In 1998, this radical correction was modified, achieving satisfactory treatment effect in children with HD, after which the operation was introduced into China and widely applied [3].

In anal modified Soave radical correction, the abdomen does not need to be opened with less blood loss, shorter operation time and rapid postoperative recovery, so it has replaced the modified Duhamel radical correction as a new treatment method for HD.

In this study, 40 child patients were selected as objects of study to investigate the long-term complications of modified Soave radical correction in the treatment of HD and its influences on life quality. It is now reported as follows.

\section{Materials and Methods}

\section{General materials}

A total of 50 child patients with Hirschsprung's disease treated in our hospital from January 2014 to September 2017 were selected as the objects of study, including 38 males and 12 females aged 0.5-49 months old with an average of $19.74 \pm$ 3.52 months old; the body weight was $4-17 \mathrm{~kg}$ with an average of $14.87 \pm 4.85 \mathrm{~kg}$. In addition, 50 normal children receiving physical examination in our hospital during the same period were selected as control group, including 36 males and 14 females aged 72 months with an average body weight of 21.45 $\pm 2.31 \mathrm{~kg}$. Relevant indicators were compared.

\section{Treatment methods}

Before operation, the liver function, blood routine and other examinations were performed, the anemia and hypoproteinemia in children were corrected, and the water and electrolyte balance was maintained. At 5-7 d before operation, reverse-flow intestinal lavage was performed, retention enema via metrodozole was conducted at $3 \mathrm{~d}$ before operation, and patients were fasted at $24 \mathrm{~h}$ before operation. After general anesthesia via tracheal intubation or epidural anesthesia, the anus of children was expanded under a lithotomy position. The anal skin was sutured using 8 drawn wires in radial pattern, and 
the anus of children was pulled to fully expose the rectal dentate line; epinephrine saline (1:200000) was injected via the rectal mucosa. The anorectum mucous layer was cut at $0.5-1$ $\mathrm{cm}$ above the inferior rectal wall and at $2-2.5 \mathrm{~cm}$ above the anterior rectal wall in the high-front low-back form. Then the edge of proximal incision was sutured using 6-8 drawn lines. The drawn lines were pulled to raise the rectal mucosa up for 3-5 cm. A 5-6 cm mucosal sheath was built and separated above the peritonic reflection. The rectal muscle sheath was cut open annularly into the abdominal cavity. The drawn line was further pulled and separated down to the bottom to fully expose the inferior intestinal wall. The narrow intestinal canal and expanded intestinal canal were separated and ligated. If the intestinal canal to be expanded was too long, the nonhypertrophic intestinal canal with expansion characteristics could be retained until the bowel morphology and intestinal wall color were normal and the texture was soft. The colon lesion was pulled out from the rectal muscle sheath, and then resected. A V-shape incision was made from muscle sheath of posterior rectal wall to the $0.5 \mathrm{~cm}$ above the teeth straight line; the tissue lesion was rapidly frozen to be tested. The colon seromuscular layer and rectal muscular sheath were intermittently anastomosed, and the colon and rectal mucosa were intermittently anastomosed to build the high-front lowback anastomotic stoma. The anastomotic stoma was fixed, vaseline gauze was inserted into the anus, and the anal canal was retained for $72 \mathrm{~h}$. After operation, antibiotics were applied continuously for 5-7 d, followed by fasting at $2 \mathrm{~d}$ and nutrition support. At $3 \mathrm{~d}$ after operation, patients could have liquid diet. At 2 weeks after operation, the anus was expanded for $15 \mathrm{~min} /$ time, 1 time/d for 3-6 months.

\section{Observation indexes}

Patients were followed up for 3 months- 6 y after operation to observe the long-term complications, life quality and anus function of children.

TACQOL scale was used for life quality score, including 7 dimensions (physical function, motor function, self-care ability, cognitive ability, communicative competence, positive emotion and negative emotion) with 8 items in each dimension. The total score of the first 5 dimensions was 32 points, while that of the latter two dimensions was 16 points; the higher the score was, the higher the life quality would be. The specific scoring methods are as follows: investigators raised the question and parents answered it, based on which the score was calculated. For example, "Does your child have trouble in access to toilets alone?", 4 points for "no"; if it occasionally and often occurred, investigators continued to ask "How about his mood faced with difficulty?", 3 points for "Good", 2 points for "Not good", 1 point for "Bad", and 0 point for "Very bad". All of 50 children were followed up and scored at the age of 6 , and there was no missing case.

HD anal function criteria proposed by Reding was used for anal function scoring [4]: (1) Defecation frequency: 2 points: $\geq$ 1 time a d: 1 point: $\geq 3$ times a week; 0 points: $<3$ times a week; (2) Abdominal distension: 2 points: no; 1 point: occasionally; 0 points: often; (3) Soiling and incontinence: 4 points: no; 3 points: $<3$ times a week; 2 points: $\geq 3$ times a week; 1 point: incontinence; 0 point: complete incontinence and soiling. The overall score was 8 points: $7-8$ points indicated the excellent, 5-6 points indicated the good, 3-4 points indicated the general, 0-2 points indicated the poor.

\section{Statistical methods}

SPSS20.0 was used for data statistics, analysis and processing; measurement data were presented as $(\overline{\mathrm{x}} \pm \mathrm{s})$ and $\mathrm{t}$-test was used. Enumeration data were presented as case (n), and $\chi^{2}$ test was used. $\mathrm{p}<0.05$ suggested that the difference was statistically significant.

\section{Results}

\section{Long-term complications of child patients after operation}

In this study, there was a total of 1 case of intestinal obstruction; 1 case of constipation that was relieved after anal dilatation and gradually recovered; 2 cases of soiling, 2 cases of urinary dysfunction that was improved after defecation training and biofeedback treatment; 1 case of diarrhoea that was cured after symptomatic treatment; 1 case of Hirschsprung's disease and aganglionosis via barium enema and rectal mucosal biopsy at 3 months after operation that received radical correction. There were a total of 8 cases with the incidence rate of $16 \%$.

\section{Comparisons of TCCQOL scores between 6-year-old child patients and 6-year-old normal children}

There was no significant difference in self-care ability between child patients and normal children $(\mathrm{P}>0.05)$; the motor function of child patients was significantly different from that of normal children $(\mathrm{P}<0.05)$; the physical function, cognitive ability, communicative ability, positive emotion, negative emotion and total score of child patients were significantly different from those of normal children $(\mathrm{P}<0.01)$ (Table 1$)$.

Table 1. Comparisons of TCCQOL scores between child patients and normal children (n (\%)).

\begin{tabular}{|c|c|c|c|c|c|c|c|c|c|}
\hline Group & & $\begin{array}{l}\text { Physical } \\
\text { function }\end{array}$ & Motor function & Self-care ability & $\begin{array}{l}\text { Cognitive } \\
\text { ability }\end{array}$ & $\begin{array}{l}\text { Communicative } \\
\text { ability }\end{array}$ & Positive emotion & $\begin{array}{l}\text { Negative } \\
\text { emotion }\end{array}$ & $\begin{array}{l}\text { Total } \\
\text { score }\end{array}$ \\
\hline $\begin{array}{l}\text { Child } \\
(n=50)\end{array}$ & patients & $29.54 \pm 3.38$ & $31.46 \pm 1.25$ & $31.84 \pm 0.56$ & $30.15 \pm 0.63$ & $30.47 \pm 1.58$ & $13.29 \pm 1.76$ & $12.25 \pm 2.49$ & $\begin{array}{l}168.74 \\
9.27\end{array}$ \\
\hline
\end{tabular}




\begin{tabular}{|c|c|c|c|c|c|c|c|c|c|}
\hline $\begin{array}{l}\text { Control } \\
(n=50)\end{array}$ & group & $31.76 \pm 0.85$ & $31.93 \pm 0.47$ & $31.98 \pm 0.39$ & $31.79 \pm 0.34$ & $31.76 \pm 0.43$ & $15.85 \pm 0.52$ & $15.92 \pm 0.46$ & $\begin{array}{l}189.57 \\
3.78\end{array}$ \\
\hline$t$ & & 4.504 & 2.489 & 1.451 & 4.373 & 5.571 & 9.864 & 10.249 & 14.713 \\
\hline$P$ & & 0 & 0.015 & 0.15 & 0 & 0 & 0 & 0 & 0 \\
\hline
\end{tabular}

\section{Anus function score of child patients}

In terms of anus function, there were 35 excellent cases, 8 good cases, and 7 general cases with the excellent-good rate of $86 \%$.

\section{Discussion}

HD is mostly caused by heredity. In the process of embryonic neurodevelopment, the impaired development of intestinal neurons occurs, leading to the loss of ganglion cells at the distal intestine, affecting the normal development of intestinal contractile function in children, ultimately producing Hirschsprung's disease [5]. If not treated in time, it will be complicated with a variety of deformities [6], and surgical treatment is the only radical method of HD, which aims to remove the aganglionic intestinal canal, and pull the intestinal canal controlled by normal nerves to the anus, and then anastomose it to retain the normal function of anal sphincter and reconstruct the continuous digestive tract.

This study showed that there were a total of 8 cases of longterm postoperative complications in child patients with the incidence rate of $16 \%$. The life quality scores of child patients were still significantly different from those of normal children $(\mathrm{P}<0.05)$. The excellent-good rate of anal function was up to $86 \%$.

There are many postoperative complications of HD, and many typical long-term complications will occur. Constipation often occurs at more than 1 month after operation, which is mostly caused by intraoperative injury or ischemia-induced spasm of internal sphincter. Child patients need cleansing enema timely and conservative treatment for more than 3 months. If the symptoms are still not improved, radical surgery is performed; the lesions should be quickly frozen for pathological examination during operation. Urinary dysfunction is also related to the damage degree of sphincter; therefore, in the process of operation, the edge of mucosal incision should be selected carefully to ensure that it is at $0.5 \mathrm{~cm}$ away from the dentate line; the high incision edge should be avoided, or the too-long aganglionic segment will easily lead to postoperative constipation, diarrhoea, recurrence and other long-term complications; if the incision edge is too low, the incidence rates of complications, such as soiling and incontinence, may be increased.

TACQOL scale was designed by Vogels et al. in Leiden University. Studies have revealed that the effects of inflammatory bowel disease on life quality of children are mostly manifested in 5 dimensions, namely the physical status, motor function, self-care ability and emotion [7]. Soiling, incontinence and constipation, as three long-term complications, after operation are key incentives affecting the life quality of children, so children need long-term anal dilatation, enema and other treatment methods, leading to peculiar smell; as a result, the normal communication of children is greatly disturbed, and children will suffer from psychological and emotional problems, such as withdrawal, poor self-confidence, depression and lack of self-awareness. Such psychological problems have become the most important factors affecting postoperative life quality of children [8]. Once soiling occurs in children, it will continue till $15 \mathrm{y}$ old, which will be significantly improved after puberty, helping improve the life quality of children [9]. Defecation, anus function, mental health education, parental care and assistance are important factors of psychological problems in children [10]. Children with social psychological disorders and lower emotional scores after operation can be improved and guided from these three aspects, so as to constantly improve the psychological state of child patients and increase their confidence in long-term recovery. Modified Soave radical correction will not leave incision scars on the abdominal wall of child patients, and will promote the recovery of anus function in children. In the later treatment, the effective defecation training and biofeedback treatment for child patients can further promote the improvement and recovery of defecation function, which can produce positive chain reactions for the psychological status and life quality of child patients.

\section{Conclusion}

In the treatment of Hirschsprung's disease, modified Soave radical correction can reduce the incidence rate of postoperative long-term complications, and help shorten the recovery time of defecation function and anus function after operation, thus gradually improving children's life quality.

\section{References}

1. Coyle D, O'Donnell AM, Gillick J. Altered neurotransmitter expression profile in the ganglionic bowel in Hirschsprung's disease. J Pediatr Surg 2016; 51: 762-769.

2. Georgeson KE, Fuenfer MM, Hardin WD. Primary laparoscopic pull-through for Hirschsprung's disease in infants and children. J Pediatr Surg 1995; 30: 1017-1022.

3. L De la T-M, Ortega-Salgado JA. Transanal endorectal pull-through for Hirschsprung's disease. J Pediatr Surg 1998; 33: 1283.

4. Reding R, Goyet JDVD, Gosseye S. Hirschsprung's disease: A 20-year experience. J Pediatr Surg 1997; 32: 1221-1225. 
5. Tjaden NEB, Trainor PA. The developmental etiology and pathogenesis of Hirschsprung disease. Transl Res 2013; 162: 1-15.

6. Hirose R. Hirschsprung's disease. Springer, Japan 2016; 239-245.

7. Loonen HJ, Grootenhuis MA, Last BF. Quality of life in paediatric inflammatory bowel disease measured by a generic and a disease-specific questionnaire. Acta Paediatr 2002; 91: 348-354.

8. Hartman EE, Oort FJ, Aronson DC. Critical factors affecting quality of life of adult patients with anorectal malformations or Hirschsprung's disease. Am J Gastroenterol 2004; 99: 907.

9. Saleh W, Rasheed K, Mohaidly MA. Management of Hirschsprung's disease: a comparison of Soave's and Duhamel's pull through methods. Pediatr Surg Int 2004; 20 : 590-593.
10. Ludman L, Spitz L, Tsuji H. Hirschsprung's disease: functional and psychological follow up comparing total colonic and rectosigmoid aganglionosis. Arch Dis Child 2002; 86: 348-351.

\section{*Correspondence to}

Yajun Chen

Department of Surgery

Beijing Children's Hospital Affiliated to Capital Medical University

Beijing

PR China 